\author{
Classification \\ Physics Abstracts \\ $61.16 \mathrm{P}-68.20-73.20$
}

\title{
Theoretical analysis of tip-MgO(100) surface interactions
}

\author{
Eric Castanier and Claudine Noguera \\ Laboratoire de Physique des Solides, associé au CNRS, Université Paris Sud, 91405 Orsay, France
}

(Received July 4; accepted October 26, 1994)

\begin{abstract}
Résumé. - Nous présentons un calcul auto-cohérent des interactions entre une pointe et une surface, supposées rigides, qui représente une première approche des images obtenues en Microscopie à Force Atomique. La surface $\mathrm{MgO}(100)$ est modélisée par un agrégat immergé et la pointe par un agrégat cubique présentant diverses orientations : 1) un coin $\mathrm{Mg}$ ou $\mathrm{O}$ au-dessus de la surface, et 2) une face parallèle à cette dernière. Deux cas sont envisagés : une surface parfaitement plane et une surface rugueuse, comportant quatre atomes additionnels adsorbés. Aucune répétition périodique du système n'est supposée, ce qui évite d'introduire des interactions latérales artificielles. En fonction de la géométrie de la pointe et de la valeur de la force choisie pour imager la surface, nous discutons quelques facteurs qui influent sur la corrugation et sur la résolution d'une mesure de Microscopie à Force Atomique.
\end{abstract}

\begin{abstract}
We present a quantum self-consistent calculation of tip/insulating surface interactions, performed under the assumption of a rigid geometry, intended to be a first step in the study of images recorded in Scanning Force Microscopy. The $\mathrm{MgO}(100)$ surface is modelled by an embedded cluster and the tip by an $\mathrm{MgO}$ cube presenting various orientations: 1) an $\mathrm{Mg}$ or $\mathrm{O}$ corner on-top the surface, or 2) a face parallel to the surface. Two cases are considered: a perfectly planar surface and a rough surface with four additional adsorbed atoms. No supercell geometry is assumed which avoids spurious lateral interactions. As a function of the tip geometry and of the force value chosen to image the surface, we discuss some factors which drive the corrugation and the resolution in an Atomic Force Microscope measurement.
\end{abstract}

\section{Introduction.}

Due to the development of the Atomic Force Microscope (AFM) [1], insulating surfaces or molecules can now be imaged. Two topographic modes are mainly used: the attractive mode performed at long distances in which van der Waals forces prevail, and the repulsive mode in which the tip is in contact with the surface and experiences short-range forces. The first mode is well suited to image large objects: biological or large organic molecules, steps, large scale roughnesses ... while the second is required to attain atomic resolution [2], for atom manipulation [3] or in 
friction experiments [4]. Many questions related to the use of the microscope remain unsolved, especially those concerning the theoretical resolution, the possibility of imaging defects in a periodic structure... Answering these questions is complicated by uncertainties related to the tip shape: while, in the scanning tunneling microscope, few atoms are involved in the electron tunneling, a larger number of atoms takes part in the tip-substrate interactions in an AFM; at long distances, this happens because of the long range nature of the van der Waals forces; at short distances, several microtips may simultaneously be in contact with the surface and thus experience the vertical force or the lateral friction. Despite the lack of information on the tip shape, during the last years, improvements in the force measurement in the whole tip-surface distance range have been made [5], which brings very precious information on all the forces at work: van der Waals, electrostatic, covalent or short range. This allows in particular to check the validity of the so-called universal models which predict a simple distance dependence of the interactions in adsorption, adhesion and cohesion processes [6,7]. This should also bring information on the physics of insulating surfaces which is not as well-established as for the case of metals or semiconductors.

From a theoretical point of view, various types of AFM modelizations have been performed. Some make use of classical approaches, relying upon an estimation of two-body or three-body interactions: they include calculations of the van der Waals forces $[8,9]$, relevant for images recorded in the attractive mode, and force field methods fitted to ab initio calculations [10]; they also include molecular dynamic simulations making use of effective interactions derived from the embedded atom method [11]. Quantum approaches, which account for the bond formation between the tip and the surface at short distances have also been performed, by various methods: LDA, Hartree-Fock or semi-empirical methods [12-16]. The use of these methods is limited by two kinds of difficulties: 1 ) due to the large computing times and memories required, only a small number of atoms can be modelled: one generally overcomes this limitation by using a supercell geometry, but, as a consequence, spurious lateral interactions between neighbouring cells are produced; in addition, in most $a b$ initio calculations, tips are made of very few atoms; 2 ) at small distances and especially in the repulsive mode, the tip and substrate atoms are submitted to substantial forces which displace them from their equilibrium positions, a process which has to be taken into account in order to obtain reliable tip-surface interaction strengths; while it is true that efficient optimization geometry codes associated with quantum electronic structure calculations are now available, they are nevertheless very time consuming and to our knowledge have scarcely been applied to AFM modelizations $[12,16]$. The tip and surface distortions are generally taken into account by atomistic approaches $[15,17]$ or by the continuum elasticity theory [18].

We have not yet been able to take into account atomic displacements in the present work, although we did it in the case of clean oxide surfaces, on which we have studied the mechanisms of relaxation and rumpling [19]. We present here a study in which the first difficulty is solved: by using an embedded cluster geometry which does not require to periodically reproduce the tip and the surface, and thanks to a self-consistent semi-empirical quantum approach, we have been able to model large systems: a substrate of 48 (three layers of 16 atoms) or 108 atoms (three layers of 36 atoms) respectively for the planar and rough surfaces, and a 8 atom cubic tip. By fixing the orientation of the cube, a sharp tip may be represented by the cube with its ternary [111] axis vertical, while placing the cube parallel to the surface yields a larger contact area. In addition, in the first geometry, the role of the chemical nature of the tip on the images may be studied by considering the cube ending with an anion or a cation.

The paper is organized as follows: after a brief sketch of the numerical method in section 2, we present, in sections 3 and 4, results on the interaction energies and forces between a planar or a rough (four atoms adsorbed on top the surface) $\mathrm{MgO}(100)$ surface and the various tips and we discuss the theoretical corrugation and resolution of the AFM on an insulating surface. 


\section{The method.}

We have performed a calculation of the interaction energy between a cluster (the tip) and an embedded cluster (the substrate), with an assumed rigid geometry deduced from the bulk structure. The calculation includes electrostatic, covalent, van der Waals and short range repulsion terms in the following way: the electrostatic and quantum terms are calculated thanks to a self-consistent quantum method, working at the Hartree-Fock level for the valence electrons; the eigenstates are expanded on an atomic orbital basis set assumed to be othonormal (CNDO approximation); the details of the method have been given elsewhere [20]. The substrate cluster is embedded in a three-dimensional array of point charges, which values are taken from the results of the selfconsistent calculations performed for a clean semi-infinite slab. In addition, electrons on the right hand side of the cluster are allowed to hop on atoms located on the left hand side. The electrostatic and covalent boundary effects are minimized in that way, which is especially important for insulating surfaces.

Van der Waals interactions between pairs of atoms are added; they are assumed to vary with the interatomic distance as $1 / R^{6}$. In the case of an $\mathrm{MgO}$ tip and an $\mathrm{MgO}$ substrate considered here, the $\mathrm{O}-\mathrm{O}, \mathrm{Mg}-\mathrm{Mg}$ and $\mathrm{O}-\mathrm{Mg}$ parameters have been taken from [21]; they yield a Hamaker constant for $\mathrm{MgO}$ equal to $9.4 \times 10^{-20} \mathrm{~J}$. This value cannot be directly compared with experimental data, which are not given in the literature to our knowledge; but it is close to the mica Hamaker constant $\left(14 \times 10^{-20} \mathrm{~J}\right.$ [22]), an oxide which has an optical dielectric constant close to that of $\mathrm{MgO}$.

Nearest neighbour short range repulsion is also added in an empirical way, since the quantum calculation forgets the non-orthogonality of atomic orbitals on neighbouring sites and the exchange repulsion between inner electrons. It is assumed to follow a Lennard-Jones law $A / d^{n}$. The whole set of parameters, summarized in table I, has been fitted to reproduce the $\mathrm{MgO}$ bulk cohesive properties: cohesive energy, lattice parameter and bulk modulus; it is also consistent with the known properties of the O-O and $\mathrm{Mg}-\mathrm{Mg}$ diatomic molecules [23].

\section{Images of a planar surface.}

We first consider a planar MgO surface interacting with an $\mathrm{MgO}$ tip: this latter will be called a sharp $\mathrm{O}$ (respectively $\mathrm{Mg}$ ) tip when the cube is placed over the surface with its ternary [111] axis vertical and an oxygen (resp. Mg) atom at the apex, or it will be called a cube $\theta(\theta=0$ or $\theta=45)$ when a planar face is parallel to the surface with a rotation angle of $\theta$ with respect to the surface lattice; in the following, the lateral position of the tip is given by the lateral position of the cube center, and its vertical position with respect to the surface by the elevation of its lower atom(s).

Table I. - Parameters used in the calculation: the four left columns give the values of the hopping integrals $\beta_{i}=7.6 V_{i} / d^{2}($ din $\AA$, $\beta$ in $e V)$ for $M g$-O interactions and $\beta_{i}=\left(7.6 V_{i} / R^{2}\right) \exp -2.5(d / R-1)$ (d in $\AA$; $R=1.25 \AA$ ) for $O-O$ and $M g-M g$ interactions; $C$ is the coefficient of the Van der Waals interaction; $A$ and $n$ are the parameters involved in the short range repulsion term.

\begin{tabular}{||l|c|c|c|c||c|c|c||}
\hline$M g O$ & $V_{s s \sigma}$ & $V_{s p \sigma}$ & $V_{p p \sigma}$ & $V_{p p \pi}$ & $C$ & $A$ & $n$ \\
\hline \hline$M g-O$ & -1 & 1 & 1.4 & 0.4 & -0.963 & 89.95 & 5.655 \\
\hline$M g-M g$ & -1 & 1 & 1.4 & 0.4 & -0.0296 & 143.78 & 7 \\
\hline$O-O$ & -1 & 1 & 1.4 & 0.4 & -34.03 & 48.72 & 5.54 \\
\hline
\end{tabular}




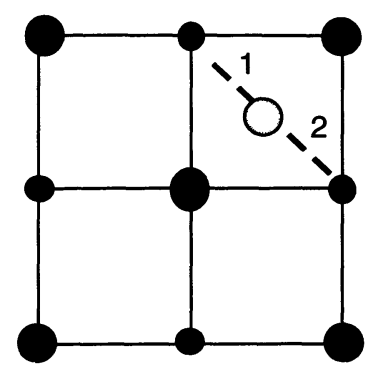

(a)

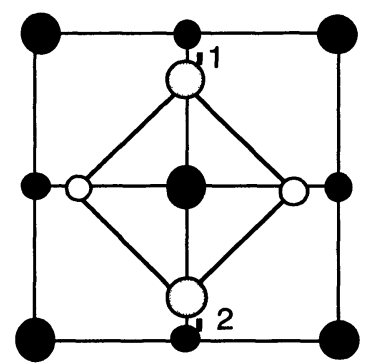

(c)

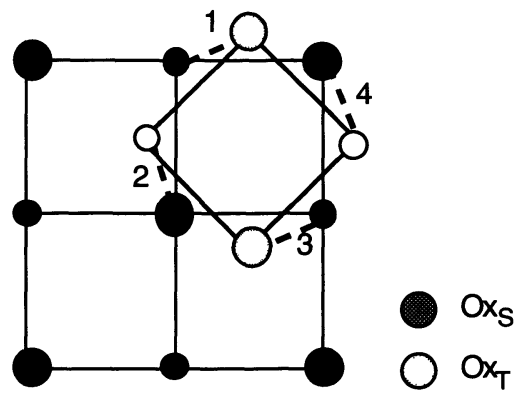

(b)

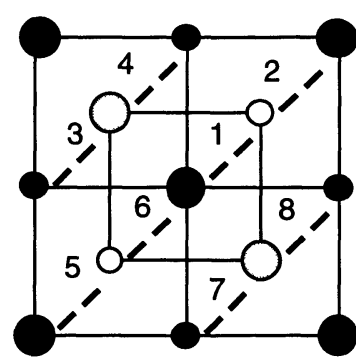

(d)

Fig. 1. - Tip-Surface bonding for various tip orientations: a sharp tip over the hollow site is associated with two $\mathrm{Mg}-\mathrm{O}$ bonds (a); Cube 45 yields two $\mathrm{Mg}-\mathrm{O}$ bonds over the hollow surface site (b) and four $\mathrm{Mg}-\mathrm{O}$ bonds over a surface atom (c); Cube 0 centred on a surface atom gives rise to eight $\mathrm{Mg}$-O bonds (d).

An embedded cluster made of 48 atoms is used here to model the surface. We will successively discuss the (adsorption) site associated with the energy minimum, the energy curve shape as a function of distance, and the surface topography in the attractive or repulsive modes.

3.1 ADSORPTION SITE. - The tip/surface geometry of lowest energy depends strongly upon the tip shape. For sharp tips, it is the hollow sites with adsorption energies respectively equal to 1.6 and $1.8 \mathrm{eV}$ for O-tips or Mg-tips, at respective elevations $z=1.6 \AA$ and $z=1.2 \AA$. For the cubes 0 and 45, the adsorption sites are respectively on-top a surface atom (oxygens and magnesiums are equivalent) $(z=2.05 \AA)$ and in the hollow sites $(z=1.8 \AA)$ with respective adsorption energies equal to $4.5 \mathrm{eV}$ and $3.8 \mathrm{eV}$. These different behaviours correspond to geometries with a maximal number of interfacial O-Mg bonds. For example, a sharp tip over the hollow surface site is associated with two Mg-O bonds (Fig. 1a) while only one would be created on-top a surface atom. The cube 45 tip located over the same hollow site yields four O-Mg bonds (Fig. 1b) to be compared to two $\mathrm{Mg}$-O bonds on-top a surface atom (Fig. 1c); the cube 0 located over a surface atom allows eight O-Mg bonds to be formed (Fig. 1d) but only 4 if it were located over the hollow site. The argument of the largest number of $\mathrm{O}-\mathrm{Mg}$ bonds for the preferred adsorption site is in agreement with what is known for adsorption processes on metal surfaces [24] and also in agreement with our study of hydroxyl group adsorption on oxide surfaces [25]. It applies each time.the bond has a large covalent character. 
These results display three important features of tip-surface interactions:

- the interaction strongly depends upon the chemical nature of the tip (oxygen or magnesium at the tip apex), as earlier noticed eg: [14];

- in the case of a cristalline tip and a cristalline substrate, the interaction energy is a function of the relative orientation of the two lattices: this is examplified here by the cube rotation. Such effect was demonstrated by the force measurement between two mica surfaces [26] and in the modelization of diamond/graphite AFM images [27];

- the interaction strength depends upon the tip size: this is easily understood for van der Waals interactions; it turns out to be also true for short range forces which depend upon the contact area.

3.2 Interaction ENERGy AS A FUnCTION OF DistanCE. - Figure 2 examplifies three behaviours displayed by the tip-surface energy curves as a function of distance. Curve (a) is obtained when a sharp O-tip is placed over a surface magnesium: it displays a long range part mainly due to attractive van der Waals interactions, a well-defined minimum at a distance close to the interatomic $\mathrm{O}-\mathrm{Mg}$ distance in $\mathrm{MgO}$, and a strongly repulsive part at short distances. Although it is slightly distorted, this curve could be compared with classical potentials for diatomic molecules.

Curve (b) corresponds to a sharp Mg-tip located on-top a surface magnesium: it involves four parts: 1) the long range attractive van der Waals behaviour, 2) a repulsive part between 2.2 and $2.7 \AA$ due to the direct electrostatic interaction, which is driven by the repulsion between the tip and surface closest magnesiums, 3) the third part is associated with the onset of lateral covalent interactions between the tip apex magnesium and the four closest surface oxygens; finally, 4) at very short distances, the short range repulsive $\mathrm{Mg}-\mathrm{Mg}$ forces prevail.

Curve (c) is obtained when a sharp Mg-tip is located over a hollow site of the surface. It involves two minima which result from the competition between the various attractive and repulsive forces already mentioned above; for example, the minimum around $z=2.1 \AA$ results from the competition between the attractive covalent energy and the repulsive electrostatic and short range terms. At smaller distances, a new attractive term, arising from the internal energy of the ions, appears, due to important charge redistributions in the tip and the substrate around the contact.

Such complicated behaviour raises several questions.

- the first point is related to the existence of several elevations of the tip corresponding to the same interacting force, which may induce tip instabilities or hysteretic behaviours, depending upon the scanning path [15]. In the following section in which we discuss surface topography, whenever such situation occurs, we will choose the highest elevation in the attractive mode and the lowest one in the repulsive mode.

- the second question is related to the applicability of universal models of adhesion to insulating systems $[6,7]$. The universal behaviour was checked by the authors for the adsorption on a metallic surface, thanks to $a b$ initio calculations and later justified by analytical arguments in the case of transition metals [28]. It relies on the recognition that two processes mainly contribute to the interaction energy: the short range repulsion and an attractive covalent term arising from the chemical bond formed between the adsorbate and the substrate and involving an electron sharing. When each of these interactions vary monotonously with the distance, the total energy curve presents a single minimum and can be scaled with a universal curve. The situation here is completely different since several types of interactions are at work, some being attractive and some repulsive: the attractive forces include the van 


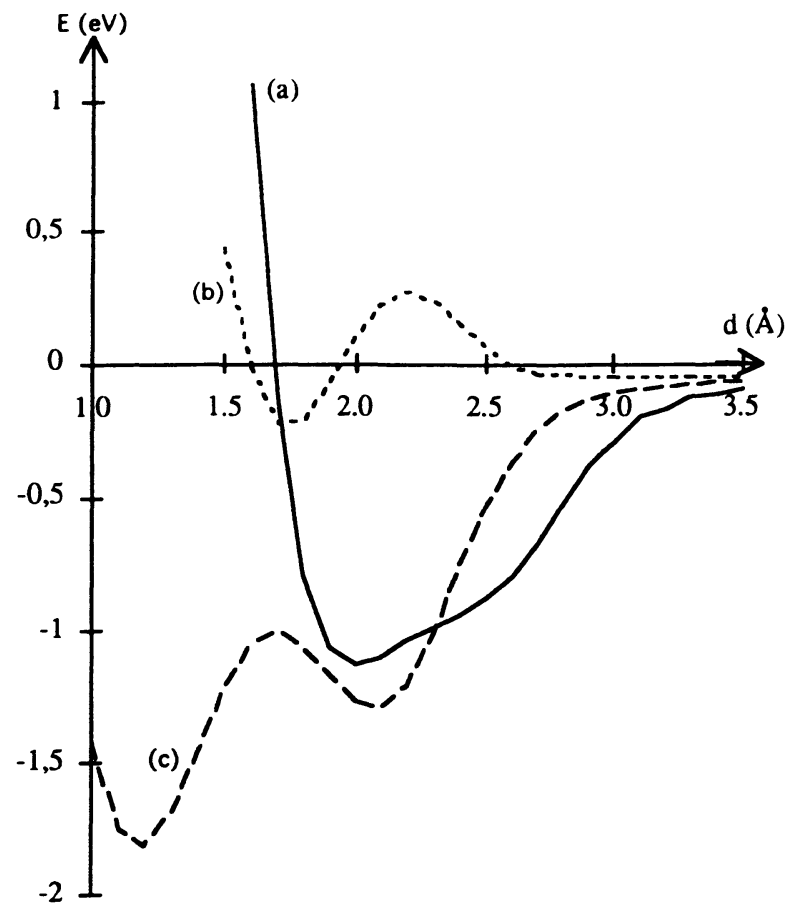

Fig. 2. - Tip-Surface interaction energy $E$ (in $\mathrm{eV}$ ) as a function of the distance $d$ between the tip apex and the surface plane (in $\AA$ ). Curve (a): Sharp O tip on-top a surface magnesium; Curve (b): Sharp Mg tip on-top a surface magnesium; Curve (c): Sharp Mg tip on-top an hollow surface site.

der Waals interactions, the $\mathrm{O}-\mathrm{Mg}$ bond formation and the $\mathrm{O}-\mathrm{Mg}$ electrostatic interaction; they may also result from electron redistributions as discussed above. The repulsive forces include the $\mathrm{O}-\mathrm{O}$ bond formation, the $\mathrm{O}-\mathrm{O}$ and $\mathrm{Mg}-\mathrm{Mg}$ electrostatic repulsion and the short range repulsion between all species. This leads to very complicated behaviours, as examplified by Figure 2, which are likely to exist in most cases where ionic materials are involved. Then, universal models are not expected to apply.

3.3 SURFACE TOPOGRAPHY . - Table II displays the tip elevation in a constant force scan over four high symmetry points of the surface cell: an oxygen, a magnesium and the two hollow sites, which are inequivalent when scanned by a cube tip. Three distinct interacting forces are considered: $F=2 \mathrm{nN}$ and $F=0.9 \mathrm{nN}$, in the repulsive regime, and $F=-2 \mathrm{nN}$ in the attractive mode. Several features are worth noticing:

- atoms do not appear systematically as protrusions in the images: for example, surface oxygens look as protrusions in the repulsive mode and hollows in the attractive mode, when scanned with a sharp O-tip;

- atoms which appear as the highest protrusions with a sharp tip may not do so with a cube-tip (eg surface oxygens at $F=0.9 \mathrm{nN}$ imaged by a sharp tip or a cube 45);

- oxygens and magnesiums cannot be discriminated on the images: depending upon the tip shape and the nature of the apex atom, depending also upon the scanning force, one or the 


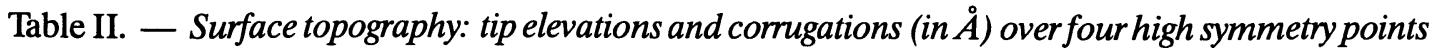
of the surface: on-top a surface oxygen, on-top a surface magnesium and over the two inequivalent hollow positions, for various tip shapes (see text) and for three different interaction forces: $F=2 n N$, $F=0.9 n N$ and $F=-2 n N$.

\begin{tabular}{|c|c|c|c|c|}
\hline & Sharp 0 & Sharp $\mathrm{Mg}$ & Cube 0 & Cube 45 \\
\hline \multicolumn{5}{|l|}{$F=2 n N$} \\
\hline$O x$ & 2.07 & 1.89 & 2 & 1.85 \\
\hline$M g$ & 1.92 & 1.69 & 2 & 2.05 \\
\hline hollow 1 & 1.52 & 1.11 & 2.08 & 1.81 \\
\hline hollow 2 & 1.52 & 1.11 & 1.98 & 1.81 \\
\hline corrugation & 0.55 & 0.78 & 0.25 & 0.25 \\
\hline \multicolumn{5}{|l|}{$F=0.9 n N$} \\
\hline$O x$ & 2.11 & 1.93 & 2.03 & 1.90 \\
\hline$M g$ & 1.95 & 1.72 & 2.03 & 2.08 \\
\hline hollow 1 & 1.55 & 1.15 & 2.11 & 1.82 \\
\hline hollow 2 & 1.55 & 1.15 & 2.0 & 1.82 \\
\hline corrugation & 0.56 & 1.17 & 0.26 & 0.26 \\
\hline \multicolumn{5}{|l|}{$F=-2 n N$} \\
\hline$O x$ & 2.25 & 2.91 & 2.86 & 3.0 \\
\hline$M g$ & 2.89 & 2.04 & 2.86 & 3.02 \\
\hline hollow 1 & 2.61 & 2.64 & 2.25 & 2.94 \\
\hline hollow 2 & 2.61 & 2.64 & 3.19 & 2.94 \\
\hline corrugation & 0.64 & 0.87 & 0.94 & 0.08 \\
\hline
\end{tabular}

other type of atom appears as the largest protrusion;

- the symmetry of the image results from a convolution of the surface periodicity and the tip symmetry;

- the surface corrugation, which is equal to the height difference between the highest protrusion and the deepest hollow, is large in the force range chosen here. Since the extrema do not necessarily correspond to atoms, but may be at hollow sites or other points in the surface cell, the value of the corrugation is not a measure of the atomic resolution; 
- the corrugation is generally larger with a sharp tip than with a cube tip; in this latter case, the summation over the atoms at the tip apex, induces an averaging of the forces which softens the differences between inequivalent points of the surface cell;

- the surface corrugation is sensitive to the rotation of the tip;

- the corrugations obtained for $F=-2 \mathrm{nN}$ are large because they correspond to an intermediate distance range in which covalent interactions or direct electrostatic interactions between the tip and the surface prevail over the van der Waals forces.

\section{Images of a surface roughness.}

We have performed calculations of the tip-surface interactions in the presence of a surface roughness. This roughness is modelled by two oxygens and two magnesiums adsorbed at missing lattice positions, in a square geometry, over a flat MgO cluster involving 108 atoms (Fig. 3). The larger cluster size was chosen in order to allow a surface scan on and around the defect, while still keeping the computing time into reasonable limits.

Figures 4 and 5 display the surface topography in the repulsive mode $(F=2 \mathrm{nN})$ obtained with a sharp O-tip and with a cube 0 . The flat surface corrugation is recovered on the extreme edges of the figures when the smallest lateral distance between the tip and the roughness is of the order of once or twice the $\mathrm{Mg}-\mathrm{O}$ interatomic distance in $\mathrm{MgO}$, a separation for which direct covalent, electrostatic and short range interactions get weaker. On-top the roughness, the image is very similar, although not completely equivalent because of lacking second neighbour interactions, to what is obtained on the flat surface.

A comparison between figures 4 and 5 reveals the importance of the tip shape on the images. These latter may be roughly interpreted as due to the convolution of the tip and defect shapes; the image obtained with a sharp tip displays an asymmetric shape, arising from the ternary symmetry of the tip. This was also true on the flat surface, but in a much less pronounced way, because of the weakness of the interactions between the surface and the atoms not located at the tip apex.

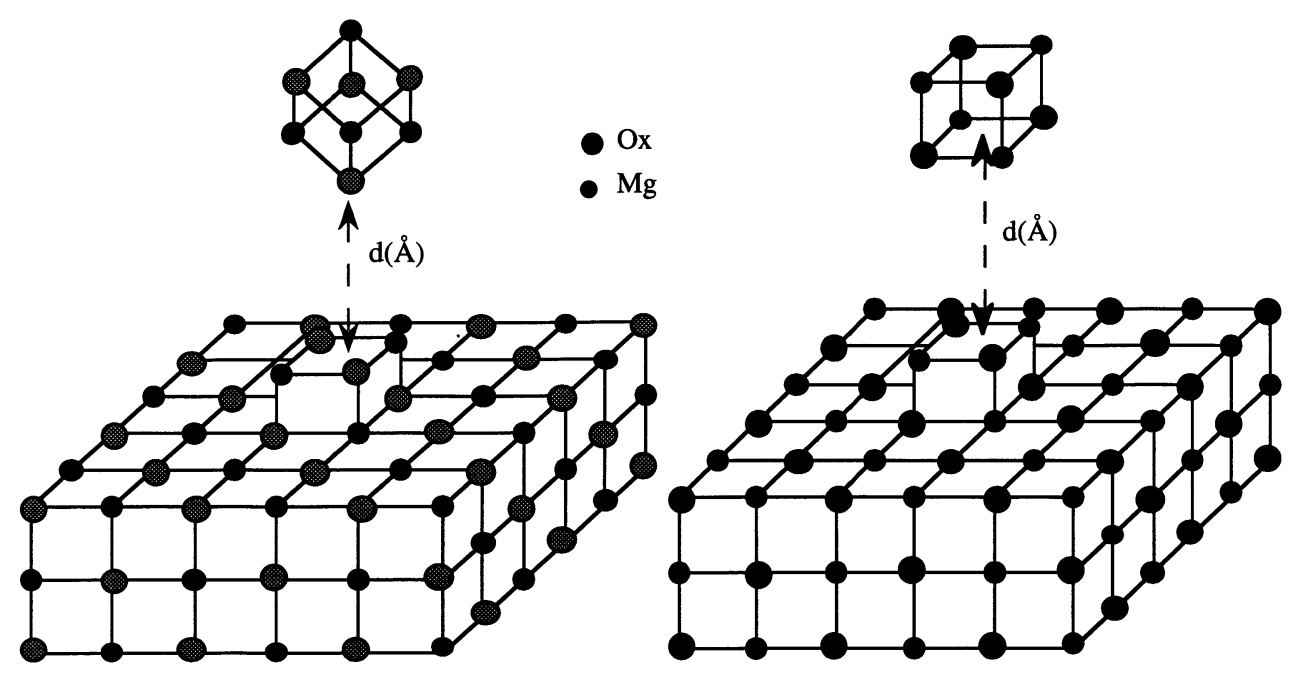

Fig. 3. - Tip-surface geometries in the modelization of a surface roughness, with a sharp tip (left) and a cube tip (right). 


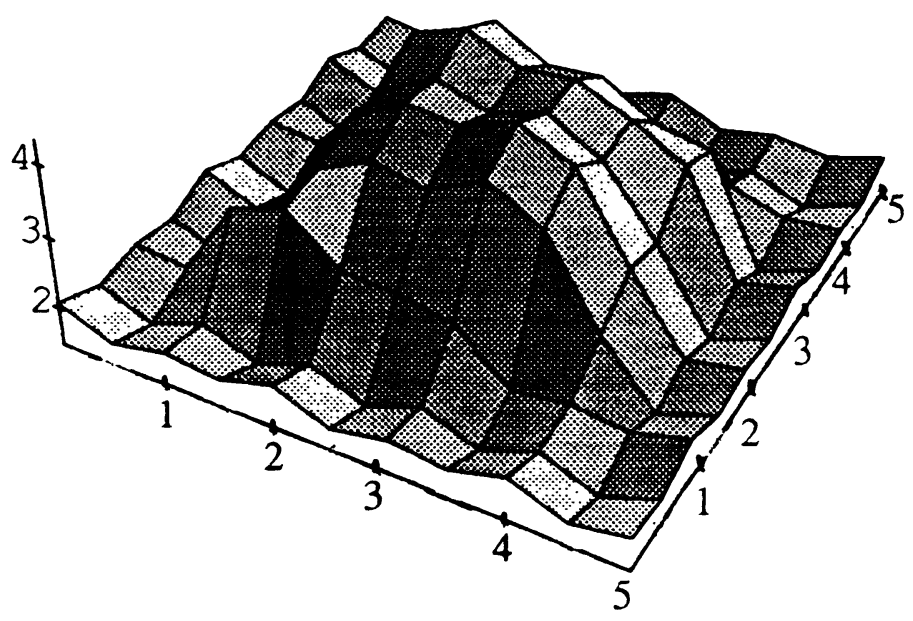

Fig. 4. - Topography of the roughness on $\mathrm{MgO}(100)$ imaged by a sharp $\mathrm{O}$ tip at $F=2 \mathrm{nN}$. Horizontal coordinates are in units of the $\mathrm{Mg}-\mathrm{O}$ interatomic distance $(2.1 \AA)$; vertical coordinates in $\AA$.

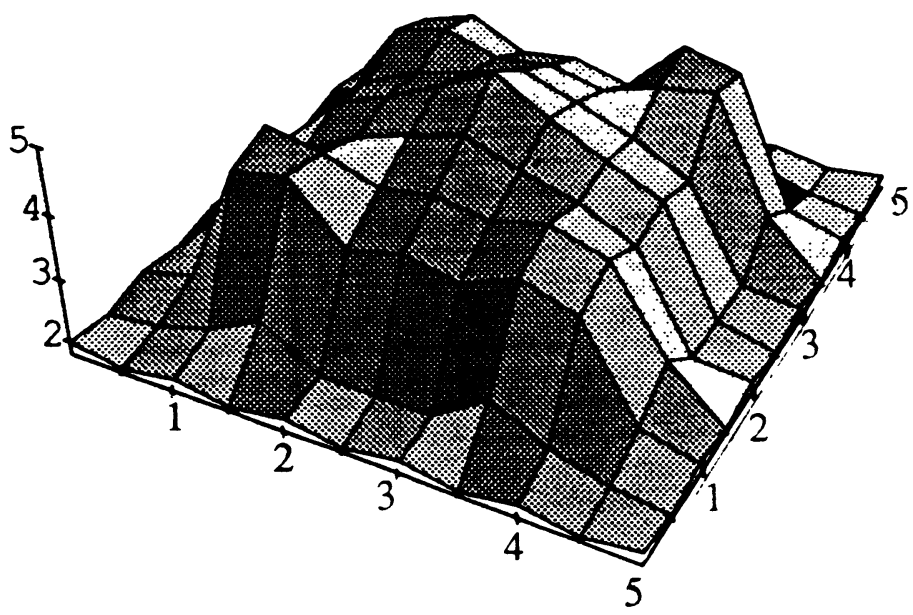

Fig. 5. - Topography of the roughness on $\mathrm{MgO}(100)$ imaged by a cube 0 tip at $F=2 \mathrm{nN}$. Horizontal coordinates are in units of the $\mathrm{Mg}-\mathrm{O}$ interatomic distance $(2.1 \AA)$; vertical coordinates in $\AA$.

On the contrary, here, some of these atoms come in direct contact with the surface protrusion and thus play a non-negligible role. The lateral sides of the roughness do not present a vertical but rather a trapezoidal shape due to the tetrahedral shape of the tip apex.

With the cube tip, on the other hand, the protrusion keeps its quadratic symmetry, and its cubic shape. Nevertheless its apparent lateral size is about twice larger than the actual one, along both directions. Its sides are steeper than when imaged by the sharp tip and its height is larger. The detailed shape of the four adsorbed atoms is sensitive to the tip rotation: the hollow site which appears here as a protrusion would be transformed into a well if the tip were rotated by 90 degrees. Despite some minor features, the overall image gives a good representation of the roughness because the short range repulsive forces prevail. The conclusion could be drastically 
different in the attractive mode at intermediate distances.

Although we have not explored larger tip sizes, this study stresses that the quality of a defect image depends upon the relative sizes and curvature radii of the tip and the defect. For those considered here, the defect is clearly seen although it might be difficult, from the image alone, to deduce its exact nature and geometry.

\section{Conclusion.}

Thanks to a quantum energy calculation, we have studied the interaction energies and forces between an $\mathrm{MgO}$ tip and an $\mathrm{MgO}$ substrate, assuming a rigid geometry. We have been able to discuss the distance dependence of the interaction for different tip shapes in terms of the competition between several attractive or repulsive elementary interactions: van der Waals, short range repulsion, direct electrostatic charge-charge interaction and covalent energy. We have shown that in most cases, this leads to non-universal behaviours of the interaction.

We have discussed the surface corrugation as a function of the applied force and as a function of the tip chemical nature, shape and orientation. In the distance range chosen here, we find that the surface corrugation is large, typically a few tenths of Ångstroems. Nevertheless, it is not generally possible to assign bumps and hollows, in the images, to atoms of different chemical nature in the unit cell. This proves the inadequacy of the AFM for chemical recognition of atoms, even for good candidates such as $\mathrm{MgO}$, which present a large electronegativity and ionic radius difference between its constituents.

The modelization of a surface roughness in the repulsive mode that we have made confirms the qualitative arguments which state that the images appear as the convolution of the tip and defect shapes. Further analysis in other force ranges is currently under study.

\section{References}

[1] Binnig G., Quate C.F., Gerber C., Phys. Rev. Lett. 56 (1986) 930.

[2] Ohnesorge F., Binnig G., Science 260 (1993) 1451.

[3] Lyo I.W., Avouris P., Science 253 (1991) 109.

[4] Mate C.M., McClelland G.M., Erlandsson R., Chiang S., Phys. Rev. Lett. 59 (1987) 1942.

[5] Gauthier-Manuel B., Europhys. Lett. 17 (1992) 195.

[6] Rose H., Smith J.R., Ferrante J., Phys. Rev. B28 (1983) 1835.

[7] Banerjea A., Smith J.R., Ferrante J., J. Phys.: Condens. Matter 2 (1990) 8841.

[8] Hartmann U., in Scanning tunneling Microscopy III (1993), Springer Series in Surface Science 29, Wiesendanger R. and Guntherodt H.J. Eds. (Springer Verlag) 293.

[9] Girard C., Phys. Rev. B43 (1991) 8822.

[10] Tekman E., Ciraci S., J. Phys. : Condens. Matter 3 (1991) 2613.

[11] Landman U., Luedtke W.D., in Scanning tunneling Microscopy III (1993), Springer Series in Surface Science 29, Wiesendanger R. and Guntherodt H.J. Eds. (Springer Verlag) 207.

[12] Ramos M.M.D., Sutton A.P., Stoneham A.M.,.J. Phys.: Condens. Matter 3 (1991) S127; ibid J. Phys.: Condens. Matter 5 (1993) 2849.

[13] Ciraci S., Baratoff A., Batra I.P., Phys. Rev. B41 (1990) 2763; Ciraci S., Tekman E., Baratoff A., Batra I.P., Phys. Rev. B46 (1992) 10411.

[14] Kotomin E., Shluger A., Causà M., Dovesi R., Ricca F., Surf. Sci. 232 (1990) 399; Shluger A., Pisani C., Roetti C., Orlando R., J. Vac. Sci. Technol. A8 (1990) 3967. 
[15] Shluger A.L., Rohl A.L., Gay D.H., Williams R.T., J. Phys.: Condens. Matter 6 (1994) 1825.

[16] Shluger A.L., Wilson R.M., Williams R.T., Phys. Rev. B49 (1994) 4915.

[17] Abraham F.F., Batra I.P., Ciraci S., Phys. Rev. Lett. 60 (1988) 1314.

[18] Zhong W., Overney G., Tomanek D., Europhys. Lett. 15 (1991) 49.

[19] Goniakowski J., Noguera C., accepted to Surf. Sci. (1994).

[20] Goniakowski J., Russo S., Noguera C., Surf. Sci. 284 (1993) 315.

[21] Girard C., Maghezzi S., Vigoureux J.M., Chem. Phys. 136 (1989) 47.

[22] Israelachvili J.N., Intermolecular and surface forces (1985), Academic Press.

[23] Huber K.P., Herzberg G. Molecular Spectra and Molecular Structure Constants of Diatomic molecules IV (Van Nostrand Reinheld Company).

[24] Desjonquères, M.C., Spanjaard D., Concepts in Surface Physics, Springer Ser. Surf. Sci. 30 (Springer Verlag, 1993).

[25] Goniakowski J., Noguera C., in Electron Processes at Surfaces, Makoshi K., Ilisca E. Eds. (World Scientific; under press).

[26] McGuiggan P.M., Israelachvili J.N., Chem. Phys. Lett. 149 (1988) 469.

[27] Tang H., Joachim C., Devillers J., Surf. Sci. 291 (1993) 439.

[28] Spanjaard D., Desjonquères M.C., Phys. Rev. B30 (1984) 4822. 